\title{
Traumatisierungen und Posttraumatische Störungen bei Patienten mit Erkrankungen aus dem schizophrenen Formenkreis
}

Ingo Schäfer ${ }^{1}$, Francisco Jose Eiroa-Orosa ${ }^{2}$, Katrin Schröder ${ }^{1}$, Timo Harfst ${ }^{3}$, Volkmar Aderhold 4

${ }^{1}$ Klinik für Psychiatrie und Psychotherapie, Universitätsklinikum Hamburg-Eppendorf, Hamburg

${ }^{2}$ School of Psychology, University of East London

${ }^{3}$ Bundespsychotherapeutenkammer, Berlin

${ }^{4}$ Institut für Sozialpsychiatrie, Ernst-Moritz-Arndt-Universität Greifswald, Greifswald

Korrespondenzadresse:

PD Dr. Ingo Schäfer, MPH

Klinik für Psychiatrie und Psychotherapie

Universitätsklinikum Hamburg-Eppendorf

Martinistr. 52

20246 Hamburg

Tel.: $\quad 040-7410-59290$

Email: i.schaefer@uke.de 


\section{Zusammenfassung}

Hintergrund: Internationale Studien zeigen, dass Patienten mit Störungen aus dem schizophrenen Formenkreis häufig Gewalt ausgesetzt waren und komorbide Posttraumatische Belastungsstörungen (PTBS) aufweisen können. Allerdings liegen aus dem deutschsprachigen Raum bislang kaum Studien vor.

Ziel der Arbeit: Das Ziel der vorliegenden Untersuchung war es, bei stationär behandelten Patienten einer deutschen Universitätsklinik die Prävalenz von Gewalterfahrungen in verschiedenen Lebensphasen und komorbiden PTBS zu bestimmen.

Methodik: Bei einer Stichprobe von N=145 konsekutiv stationär aufgenommenen Patientinnen und Patienten mit Störungen aus dem schizophrenen Formenkreis (67\% männlich) wurden anhand des Strukturierten Traumainterview (STI) interpersonelle Gewalterfahrungen und anhand des Strukturierten Klinischen Interviews für DSM-IV (SKID) komorbide PTBS erhoben.

Ergebnisse: Sexuelle Gewalt im Alter von unter 16 Jahren wurden von 17\% berichtet (Frauen: 27\%; Männer: 12\%). Etwa ein Drittel (32\%) berichtete körperliche Gewalt durch elterliche Bezugspersonen (Frauen: 38\%; Männer: 29\%). Mindestens eine Form früher Gewalt (sexuell oder körperlich) berichtete die Hälfte der Frauen (48\%) und jeder dritte Mann (34\%). Negative sexuelle Erfahrungen im späteren Leben berichteten 17\%, körperliche Gewalt 38\% und mindestens eine Form $48 \%$ der Befragten. Insgesamt berichteten zwei Drittel (66\%) Gewalterfahrungen im Leben. Die Punktprävalenz der PTBS betrug 12\%.

Diskussion: In der vorliegenden Untersuchung wurden die berichteten Raten von interpersonellen Gewalterfahrungen und komorbiden PTBS bei Patientinnen und Patienten mit Störungen aus dem schizophrenen Formenkreis in einer deutschen Stichprobe bestätigt werden. Gewalterfahrungen und ihre Folgen sollten deshalb routinemäßig erhoben und das gesamte Spektrum traumatherapeutischer Ansätze in die Behandlung dieser Patientengruppe integriert werden.

\section{Schlüsselwörter}

Trauma, Gewalt, Posttraumatische Belastungsstörung, Schizophrenie, Psychose 


\section{Abstract}

Background: The findings of international studies suggest high rates of interpersonal violence and posttraumatic stress disorder (PTSD) among patients with schizophrenia spectrum disorders. Only few studies, however, have been conducted in the German-speaking countries so far.

Objectives: The aim of study was to determine the prevalence of lifetime experiences of interpersonal violence and comorbid PTSD among inpatients in a German university hospital.

Method: In $\mathrm{N}=145$ consecutively admitted patients with schizophrenia spectrum disorders (67\% male), we used the Structured Traumainterview (STI) to assess experiences of interpersonal violence and the Structured Interview for DSM-IV (SCID) to assess comorbid PTSD.

Results: Sexual violence under the age of 16 was reported by $17 \%$ of the patients (women: 27\%; men: 12\%). About one third (32\%) reported physical violence by parental figures (women: 38\%; men: 29\%). At least one form of early violence (sexual or physical) was reported by half of the women (48\%) and one third of the men (34\%). Negative sexual experiences later in life were reported by $17 \%$, physical violence by $38 \%$, and at least one of these forms by $48 \%$ of the patients. In total, on third of all patients $(66 \%)$ reported experiences of violence during their lifetime. The prevalence of acute PTSD was $12 \%$.

Conclusions: The presents study confirmed the high rates of experiences of interpersonal violence and comorbid PTSD in a German sample of patients with schizophrenia spectrum disorders. Violence and it's consequences should therefore be routinely assessed and the full spectrum of trauma-specific therapies should be integrated in the treatment of this group of patients.

\section{Keywords}

Trauma, Violence, Posttraumatic Stress Disorder, Schizophrenia, Psychosis 
Traumatische Erfahrungen in der Kindheit, wie Erfahrungen sexueller und körperlicher Gewalt, werden bei psychotischen Erkrankungen als ätiologisch bedeutsamer Faktor angesehen und sind für deren Verlauf von Bedeutung. Neben komorbiden Posttraumatischen Belastungsstörungen können weitere, komplexe Traumafolgen das klinische Bild bei betroffenen Patientinnen und Patienten erschweren, werden aber bislang zu selten in Psychosetherapien berücksichtigt. Während diese Zusammenhänge gut belegt sind, liegen aus dem deutschsprachigen Raum bislang kaum Zahlen zur Prävalenz von Gewalterfahrungen und Posttraumatischen Störungen bei Patienten mit Psychosen aus dem schizophrenen Formenkreis vor.

\section{Hintergrund}

Internationale Studien weisen darauf hin, dass Patienten mit psychotischen Störungen in ihrer Biographie häufig Gewalterfahrungen und anderen potenziellen Traumatisierungen ausgesetzt waren. Morgan und Fisher [18] kamen in einer Übersicht über 20 Studien zu dem Ergebnis, dass sexuelle Gewalt in der Kindheit im Mittel von $42 \%$ der weiblichen und $28 \%$ der männlichen Patienten mit psychotischen Erkrankungen berichtet wurde, körperliche Misshandlung von 35\% bzw. 38\%. Mindestens eine Form früher Gewalt berichteten jeweils 50\% der Patientinnen und Patienten. Eine neuere Übersicht [4] kam über beide Geschlechter hinweg zu ähnlichen Ergebnissen in Bezug auf körperliche Gewalt (39\%). Die Häufigkeit sexueller Gewalt in der Kindheit wurde in dieser Analyse dagegen etwas geringer eingeschätzt (26\%), wobei weibliches Geschlecht ebenfalls mit einer höheren Prävalenz assoziiert war. Weitere Formen früher interpersoneller Traumatisierungen, wie emotionale Misshandlung und Vernachlässigung, werden von bis zu $65 \%$ der Patientinnen und Patienten mit Psychosen berichtet (z.B. [25; 29]).

Auch im Erwachsenenalter sind Patienten mit schweren psychischen Erkrankungen häufig Gewalt ausgesetzt. So berichteten in einer Untersuchung aus Großbritannien 23\% der befragten Psychosepatienten in den zurückliegenden zwei Jahren Übergriffen mit Körperkontakt ausgesetzt gewesen zu sein [7]. In einer schwedischen Studie betrug diese Rate bezogen auf das letzte Jahr 20\% [2]. Sexuelle Übergriffe innerhalb des letzten Jahres berichteten in dieser Studie 12\% der Frauen und 3\% der Männer. Die Häufigkeit von Gewalterfahrungen im Erwachsenenalter wird dabei 
durch verschiedene Faktoren beeinflusst, wie die Schwere der Erkrankung, zusätzliche Substanzprobleme oder die soziale Situation der Patientinnen und Patienten [2; 7].

Besonders Gewalterfahrungen in Kindheit und Jugend sind bei Psychosepatienten mit zusätzlichen klinischen Problemen assoziiert, wie sie auch bei anderen Patientengruppen mit frühen Traumatisierungen anzutreffen sind. Dies betrifft etwa mehr depressive Symptome und Ängstlichkeit [17; 24], mehr Suizidversuche und selbstverletzendes Verhalten [6; 24], mehr Substanzmissbrauch [6] und mehr Probleme in nahen Beziehungen [16]. Verschiedene Studien berichteten auch von stärker eingeschränkten kognitiven Funktionen bei Patienten mit frühen Traumatisierungen, die deren Fähigkeit von therapeutischen Angeboten zu profitieren beeinträchtigen und so $\mathrm{zu}$ einer ungünstigeren Prognose beitragen könnten [25]. Neben diesen allgemeinen Folgen früher Gewalterfahrungen können auch komorbide Posttraumatische Belastungsstörungen (PTBS) vorliegen, die in der Praxis häufig unerkannt bleiben. Internationale Untersuchungen zur Prävalenz einer komorbiden PTBS bei Personen mit psychotischen Störungen berichteten Raten von $14 \%$ bis $46 \%$ (Übersicht bei [22]). Die Symptome der PTBS belasten Betroffene zusätzlich und können ihren Krankheitsverlauf dadurch verschlechtern, dass sie psychotische Symptome verstärken, zu sozialem Rückzug beitragen und das bereits erhöhte Erregungsniveau bei psychotischen Patienten weiter steigern [19].

Während die oben geschilderten Befunde nahelegen, dass Gewalterfahrungen und posttraumatische Störungen bei Patienten mit psychotischen Störungen häufig sind und sie eine wichtige Rolle für den Krankheitsverlauf spielen, können sie nicht ohne Weiteres auf den deutschsprachigen Raum bezogen werden. So bestehen bereits in Bezug auf traumatische Erfahrungen in der Allgemeinbevölkerung deutliche Unterschiede zwischen verschiedenen Ländern bzw. Studien (vgl. Glaesmer et al. in diesem Heft). Zudem weisen viele der oben zitierten Studien methodische Einschränkungen auf. So wurden oft keine strukturierten Interviews, sondern lediglich Selbstbeurteilungsinstrumente eingesetzt um Gewalterfahrungen und posttraumatische Störungen $\mathrm{zu}$ erheben, es wurden keine Angaben zum Krankheitsstadium gemacht und nicht zwischen Psychosen aus dem schizophrenen Formenkreis und affektiven Psychosen unterschieden. Das Ziel der vorliegenden 
Untersuchung war es deshalb, bei Patientinnen und Patienten, die aufgrund einer Störung aus dem schizophrenen Formenkreis in einer deutschen Universitätsklinik stationär behandelt wurden, anhand strukturierter Interviews die Prävalenzraten von Gewalterfahrungen in verschiedenen Lebensphasen und komorbiden Posttraumatischen Belastungsstörungen zu bestimmen.

\section{Methode}

\section{Stichprobe}

In die Studie wurden Patientinnen und Patienten eingeschlossen, die konsekutiv auf eine offene Schwerpunktstation für psychotische Erkrankungen der Klinik für Psychiatrie und Psychotherapie des Universitätsklinikums Hamburg-Eppendorf aufgenommen wurden. Einschlusskriterien waren die Diagnose einer Störung aus dem schizophrenen Formenkreis (ICD-10 F20-F29), ein Alter von 18 bis 65 Jahren und ausreichende deutsche Sprachkenntnisse. Ausschlusskriterien waren klinische Aspekte, die zu einer potenziellen Gefährdung im Falle einer Teilnahme führten (z.B. anhaltende akute Suizidalität) und eine Aufenthaltsdauer $\leq 3$ Tage. Von $N=283$ Patientinnen und Patienten, die im Untersuchungszeitraum die Einschlusskriterien erfüllten, entschieden sich 178 (63\%) an der Studie teilzunehmen. Davon konnten 20 nicht untersucht werden da sie die Station wieder verließen, bevor die Interviews durchgeführt werden konnten. Zwei Patienten brachen die Interviews ab, da sie sich zu stark belastet fühlten. Bei zwei weiteren Patienten wurde durch die Interviewer entschieden, die Befragung abzubrechen. Die Daten von neun Patienten wurden aufgrund widersprüchlicher Angaben später von der Analyse ausgeschlossen. Die endgültige Stichprobe umfasste somit N=145 Patientinnen und Patienten.

\section{Instrumente}

Die Diagnose einer Störung aus dem schizophrenen Formenkreis wurde mit dem MINI International Neuropsychiatric Interview gestellt (M.I.N.I.; [15]) und anhand des Psychose-Moduls des Strukturierten Klinischen Interviews für DSM-IV (SCID I; [10]) gesichert. Das Ausmaß der psychotischen Symptomatik wurde anhand der Positive and Negative Syndrome Scale (PANSS; [13]) bei Aufnahme und erneut zum Zeitpunkt der Traumadiagnostik erhoben. Gewalterfahrungen in verschiedenen Lebensabschnitten wurden mithilfe des Strukturierten Traumainterviews (STI; [8]) 
erhoben. Das STI wurde für epidemiologische Untersuchungen zu interpersoneller Gewalt entwickelt und kam bereits in zahlreichen Untersuchungen bei psychiatrischen Patienten zum Einsatz (z.B. [21; 14]). Der Schwerpunkt des Interviews liegt auf körperlichen und sexuellen Gewalterfahrungen in der Kindheit. Darüber hinaus werden Zeugenschaft von Gewalt zwischen den Eltern, frühe Trennung von elterlichen Bezugspersonen, Indikatoren einer „familiären Dysfunktion“ (etwa psychische Erkrankungen oder Suchtprobleme der Eltern) sowie sexuelle und körperliche Gewalt im Erwachsenalter erhoben. Negative sexuelle Erfahrungen vor dem 16. Lebensjahr werden handlungsbezogen erfasst, von sexuellen Berührungen bis hin zu Erfahrungen mit Penetration. Dabei wird eine „enge Definition“ zugrunde gelegt, d.h. nur Erfahrungen mit Körperkontakt werden als sexuelle Gewalt gewertet. Zusätzlich werden weitere Aspekte, etwa die Anzahl verschiedener Täter erfasst. Auch körperliche Gewalt in der Kindheit wird handlungsbezogen erfasst und als Kombination von schwerwiegenden aggressiven Handlungen durch elterliche Bezugspersonen definiert (z.B. getreten, geboxt, verbrüht oder mit Gegenständen geschlagen werden). Sexuelle und körperliche Gewalt im Erwachsenenalter wird jeweils mit einer Frage erhoben („Haben Sie später im Leben negative sexuelle Erfahrungen gemacht, bei denen Sie unter Druck gesetzt oder dazu gezwungen wurden Dinge zu tun, die Sie nicht tun wollten“ bzw. „Wurden Sie später im Leben jemals geschlagen?"). Zur Diagnostik komorbider Posttraumatischer Belastungsstörungen wurde das PTBS-Modul des Strukturierten Klinischen Interviews für DSM-IV eingesetzt (SCID I; [10]).

\section{Vorgehen bei der Datenerhebung}

Innerhalb der ersten drei Tage des stationären Aufenthaltes wurden neu aufgenommenen Patienten, die die Einschlusskriterien erfüllten, über die Studie aufgeklärt und um ihre Teilnahme gebeten. Im Falle ihres schriftlichen Einverständnisses wurden die soziodemographischen Daten der Patienten erhoben, die Diagnose einer Störung aus dem schizophrenen Formenkreis anhand des M.I.N.I. und des Psychosemoduls des SKID gesichert und ein PANSS-Interview durchgeführt. Die Traumadiagnostik erfolgte erst zu einem Zeitpunkt, zu dem die Patienten vom Behandlungsteam als ausreichend stabilisiert angesehen wurden ( $M=20,9$ Tage nach Aufnahme; SD=13,2). Zu diesem Zeitpunkt wurde erneut ein PANSS-Interview durchgeführt und es zeigte sich, dass die PANSS-Werte signifikant 
gesunken waren ( $M=67,1 \pm 14,1$ vs. $M=80,6 \pm 15,7$ bei Aufnahme; $p<0,001)$. Es folgte die Durchführung des Strukturierten Traumainterviews sowie des PTBS-Moduls des Strukturierten Klinischen Interviews für DSM-IV.

Statistische Analyse

Bei der Analyse der Daten kamen vorwiegend Methoden der deskriptiven Statistik zur Anwendung. Geschlechtsunterschiede in Bezug auf soziodemographische Daten und interpersonelle Traumatisierungen wurden anhand von Chi-Quadrat-Tests und Odds Ratios überprüft. Das Signifikanzniveau wurde auf $p<0,05$ gesetzt. Die Auswertung erfolgte anhand des "Statistical Package for the Social Sciences" (SPSS, Illinois). 


\section{Ergebnisse}

\section{Soziodemographie}

Von den insgesamt $\mathrm{N}=145$ untersuchten Patientinnen und Patienten waren $\mathrm{n}=48$ (33\%) weiblich, $n=97$ (67\%) männlich. Das mittlere Alter betrug $M=34,1$ Jahre $(S D=11,5$; range=18-65). Etwas mehr als ein Drittel $(N=56 ; 39 \%)$ hatte die Schule mit dem (Fach-)Abitur abgeschlossen, weitere $\mathrm{N}=47$ (32\%) mit der mittleren Reife. Die Hauptschule hatten $\mathrm{N}=28(19 \%)$ absolviert. Lediglich $\mathrm{N}=41$ (28\%) waren erwerbstätig und $\mathrm{N}=30(20 \%)$ befanden sich in einer festen Partnerschaft.

\section{Diagnosen und Erkrankungsdauer}

Die meisten Patientinnen und Patienten ( $\mathrm{N}=104,72 \%)$ erfültten die Diagnose einer schizophrenen Störung, $\mathrm{N}=32(22 \%)$ die einer schizoaffektiven Störung und $\mathrm{N}=9$ (6\%) wiesen andere Erkrankungen aus dem schizophrenen Formenkreis auf (z.B. anhaltende wahnhafte Störungen). Die mittlere Erkrankungsdauer betrug $M=7,8$ ( $\mathrm{SD}=9.7$ ) Jahre. Bei $\mathrm{N}=45$ (31\%) war die Erkrankung innerhalb des zurückliegenden Jahres erstmalig aufgetreten, bei $\mathrm{N}=36(25 \%)$ betrug die Erkrankungsdauer zwischen einem und 5 Jahren und $\mathrm{N}=64$ (44\%) der Patientinnen und Patienten waren bereits länger als 5 Jahre erkrankt.

\section{Prävalenz von körperlicher und sexueller Gewalt}

Im Strukturierten Traumainterview berichteten $\mathrm{N}=25 \quad(17 \%)$ der befragten Patientinnen und Patienten negative sexuelle Erfahrungen mit Körperkontakt im Alter von unter 16 Jahren (Tabelle 1). Dies betraf Frauen signifikant häufiger als Männer (27\% vs. $12 \%$; OR=2,6; $95 \%-\mathrm{Cl}=1,1-6,3)$. Bei einem Drittel der Betroffenen ( $N=9$; $36 \%$ ) handelte es sich um Erfahrungen, bei denen es auch zu Geschlechtsverkehr gekommen war. Bei der überwiegenden Mehrheit ( $\mathrm{N}=22 ; 88 \%)$ handelte es sich um wiederholte Erfahrungen und $\mathrm{N}=9$ (36\%) berichteten sexuelle Gewalt durch mehrere verschiedene Täter erlebt zu haben. Bei etwa einem Viertel $(N=6 ; 24 \%)$ stammte der Täter aus der eigenen Familie, bei etwa der Hälfte ( $N=14 ; 56 \%)$ ausschließlich aus dem extrafamiliären Bereich. Einzelne Patientinnen und Patienten $(N=3 ; 12)$ berichteten sowohl durch intra- als auch durch extrafamiliäre Täter sexuelle Gewalt erlebt zu haben. 
Knapp ein Drittel der Befragten ( $N=46 ; 32 \%)$ berichtete im Alter von unter 16 Jahren körperliche Gewalt, d.h. wiederholt massive aggressive Handlungen durch Eltern oder elterliche Bezugspersonen erlebt zu haben. Auch von körperlicher Gewalt waren Frauen häufiger betroffen als Männer (38\% vs. $29 \%)$, wobei dieser Unterschied nicht statistisch signifikant war. Von den Betroffenen berichteten $\mathrm{N}=17$ (37\%) dass die Gewalterfahrungen mit körperlichen Verletzungen einhergingen. $\mathrm{N}=32(70 \%)$ erlebten Gewalt durch den (Stief-)Vater, N=22 (48\%) wurden durch die (Stief-)Mutter misshandelt und N=11 (24\%) der Betroffenen erlebten Gewalt durch beide elterliche Bezugspersonen. Mindestens eine Form von Gewalt (sexuell oder körperlich) im Alter von unter 16 Jahren berichtete knapp die Hälfte der Frauen $(\mathrm{N}=23 ; 48 \%)$ und jeder dritte Mann $(\mathrm{N}=33 ; 34 \%)$.

- Bitte Tabelle 1 etwa hier einsetzen -

Negative sexuelle Erfahrungen nach dem 16. Lebensjahr berichteten $\mathrm{N}=24$ der Befragten (17\%). Erneut waren Frauen signifikant häufiger betroffen als Männer (29\% vs. $10 \%$; OR=3,6; 95\%-Cl=1,4-9,1). Dagegen waren von körperlicher Gewalt nach dem 16 Lebensjahr, die von $\mathrm{N}=56$ (38\%) der Befragten berichtet wurde, signifikant häufiger Männer betroffen als Frauen (44\% vs. $27 \%$; OR=2,14; $95 \%$ $\mathrm{Cl}=1,01-4,55)$. Mindestens eine Form von Gewalt im Alter von über 16 Jahren (sexuelle und/oder körperlich) berichtete jeweils knapp die Hälfte aller Patientinnen und Patienten ( $\mathrm{N}=70 ; 48 \%)$ bei relativ ausgewogenem Geschlechterverhältnis (Frauen 50\%, Männer 47\%). Wurden Gewalterfahrungen über die gesamte Lebensspanne betrachtet, so berichteten zwei Drittel aller Patientinnen und Patienten ( $N=96 ; 66 \%)$ körperliche oder sexuelle Gewalt erlebt zu haben, wobei sich in Bezug auf sexuelle Gewalt signifikante Geschlechtsunterschiede fanden, mit einer größeren Häufigkeit bei Frauen ( $48 \%$ vs. $21 \%$; $\mathrm{OR}=3,6,95 \%-\mathrm{Cl}=1,7-7,7)$.

\section{Prävalenz der Posttraumatischen Belastungsstörung (PTBS)}

Das SKID-Modul zur Posttraumatischen Belastungsstörung (PTBS) wurde bei $\mathrm{N}=121$ konsekutiven Patientinnen und Patienten durchgeführt (83\%). Die Prävalenz der PTBS betrug $12 \%(\mathrm{~N}=14)$ und war bei Frauen höher als bei Männern (17\% vs. $6 \%)$ Raten aufwiesen. Bei der Mehrzahl der Betroffenen lagen Gewalterfahrungen in der Kindheit vor $(\mathrm{N}=11 ; 79 \%)$. 


\section{Diskussion}

In der vorliegenden Untersuchung berichtete die Hälfte aller weiblichen Patientinnen mit Störungen aus dem schizophrenen Formenkreis (48\%) und ein Drittel der männlichen Patienten (34\%) sexuelle und/oder körperliche Gewalt in Kindheit und Jugend. Die Prävalenz sexueller Gewalt war mit 17\% niedriger als in verschiedenen Übersichtsarbeiten zu dieser Thematik (26\% [4], bzw. 35\% [18]). Legt man die Prävalenzraten einer repräsentativen Untersuchung der deutschen Allgemeinbevölkerung zugrunde, die eine vergleichbare Altersgrenze und Definition sexueller Gewalt wählte (Frauen: 7\%; Männer: 1,4\%; [3]), waren die Raten sexueller Gewalt in der Kindheit bei weiblichen Patientinnen dennoch etwa dreifach, bei männlichen Patienten sogar achtfach erhöht. Die Prävalenz körperlicher Gewalt in der Kindheit war mit 32\% den Befunden anderer Studien bei psychotischen Patienten ähnlicher (39\% [4], bzw. 37\% [18]) und betrug das drei- bis vierfache der Prävalenz in der deutschen Allgemeinbevölkerung (ca. [30]).

In unserer Untersuchung bestätigten sich die auch in anderen Studien berichteten signifikanten Zusammenhänge zwischen weiblichem Geschlecht und sexueller Gewalt in verschiedenen Lebensphasen, das ausgeglichene Geschlechterverhältnis in Bezug auf körperliche Gewalt in der Kindheit und die häufigeren körperlichen Gewalterfahrungen bei Männern im späteren Leben (z.B. [4]). Die Rate komorbider Posttraumatischer Belastungsstörungen war mit $12 \%$ etwas niedriger als in den meisten internationalen Studien (14\% bis $46 \%$; Übersicht bei [22]). Auch in einer deutschen Studie bei 74 Patienten mit affektiven Psychosen betrug die Prävalenz der PTBS 20\% [1]. Dennoch betrug die Häufigkeit der PTBS bei den untersuchen Patientinnen und Patienten damit das fünf- bis achtfache der Prävalenz in der deutschen Allgemeinbevölkerung (1,4\% bis $2,9 \%$; z.B. [26]).

Die verglichen mit anderen Studien niedrigeren Raten von frühen sexuellen Gewalterfahrungen und Posttraumatischen Störungen in unserer Untersuchung könnten durch unterschiedliche Faktoren zu erklären sein. Dazu zählt in erster Linie, dass eine enge Definition sexueller Gewalt gewählt und ein strukturiertes Interview eingesetzt wurde um die entsprechende Erfahrungen zu erheben. In vielen der vorherigen Studien wurden dagegen weite Definitionen gewählt, die auch Erfahrungen ohne Körperkontakt einschlossen, und Selbstbeurteilungsinstrumente 
eingesetzt, die den Befragten teilweise mehr Spielraum für eigene Interpretationen lassen. Aber auch Stichprobeneinflüsse dürften eine Rolle gespielt haben. So konnte in verschiedenen Untersuchungen gezeigt werden, dass frühe Traumatisierungen bei psychiatrischen Patienten mit der Erkrankungsschwere, mit suizidalen Handlungen und mit Zwangsmaßnahmen assoziiert sind (z.B. [6; 12]). Die Rekrutierung auf der offenen Psychosestation einer Universitätsklinik könnte vor diesem Hintergrund zu einer Verzerrung hin zu einer seltener betroffenen Stichprobe geführt haben. Dasselbe trifft auf manche Ausschlusskriterien unserer Studie zu wie anhaltende Suizidalität und eine Dauer des Aufenthaltes von weniger als vier Tagen. Die Ergebnisse stellen deshalb eine eher konservative Schätzung der Prävalenz von Gewalterfahrungen und komorbiden PTBS bei Patienten mit Störungen aus dem schizophrenen Formenkreis dar.

\section{Konsequenzen für die Behandlung}

Für betroffene Patientinnen und Patienten, in unserer Studie zwei Drittel der gesamten Stichprobe (66\%), ergeben sich wichtige klinische Konsequenzen. So sind gerade Gewalterfahrungen in Kindheit und Jugend auch bei Psychosepatienten nicht nur mit Symptomen der PTBS sondern mit weiteren, komplexen Traumafolgen assoziiert, die in den etablierten Therapien für psychotische Patientinnen und Patienten oft nicht adressiert werden. Dazu zählt beispielsweise eine allgemeine emotionale Instabilität und interpersonelle Probleme, traumaassoziierte Kognitionen, Scham- und Schuldgefühle sowie ein erhöhtes Risiko erneut Gewalt ausgesetzt zu sein (z.B. [16]). Inzwischen wurden deshalb verschiedene Therapieansätze, die einen Schwerpunkt auf diese Probleme legen speziell für Patienten mit Psychosen und anderen schweren psychischen Erkrankungen entwickelt. Dazu zählt etwa das „Trauma Recovery and Empowerment Model“ (TREM; [9]), oder ein von Mueser et al. [20] vorgelegtes Therapieprogramm. Positive Erfahrungen liegen auch mit dem „Skillstraining zur affektiven und interpersonellen Regulation“ (STAIR; [5]) vor. Dieser Behandlungsansatz, der zur Behandlung der allgemeinen Folgen früher Gewalt und Vernachlässigung entwickelt wurde, zeigt auch bei Patientinnen und Patienten mit Störungen aus dem schizophrenen Formenkreis positive Effekte [27]. 
Auch zur Behandlung der PTBS mit expositionsbasierten Verfahren liegen bei Patientinnen und Patienten mit psychotischen Erkrankungen inzwischen ermutigende Befunde vor. So legten etwa Van den Berg und Kollegen [28] eine methodisch hochwertige, kontrollierte Studie zu den Effekten von 8 Sitzungen Exposition in sensu bzw. „Eye Movement Desensitization and Reprocessing (EMDR)“ bei 155 Patientinnen und Patienten mit der Lebenszeitdiagnose einer Störung aus dem schizophrenen Formenkreis und komorbider PTBS vor. Beide Expositionsverfahren zeigten darin gute Effekte, wobei das vielleicht wichtigste Ergebnis dieser Studie war, dass die Behandlung von den Teilnehmerinnen und Teilnehmern gut toleriert wurde.

\section{Limitationen}

Bei der Interpretation der Ergebnisse müssen verschiedene Limitationen berücksichtigt werden. So stellt die retrospektive Erhebung traumatischer Erfahrungen immer einen potenziellen Kritikpunkt dar. Zudem wurde in Frage gestellt, ob Traumatisierungen und Posttraumatische Störungen auch bei Patienten mit psychotischen Störungen reliabel und valide erhoben werden können. Inzwischen wird dies jedoch durch eine wachsende Zahl von Untersuchungen gestützt (z.B. [23; 11]). Zudem wurden die Patientinnen und Patienten bewusst zu einem Zeitpunkt untersucht, zu dem sich die psychotische Symptomatik bereits reduziert hatte. Eine wesentliche Einschränkung unserer Untersuchung betrifft jedoch wie oben bereits erwähnt ihre Repräsentativität für Patienten mit Störungen aus dem schizophrenen Formenkreis im deutschen Versorgungssystem. Hier muss aufgrund des Erhebungssettings und der Ein- und Ausschlusskriterien davon ausgegangen werden, dass die berichteten Prävalenzraten eher eine Unterschätzung darstellen.

\section{Schlussfolgerung}

Ein Großteil aller Patientinnen und Patienten mit Störungen aus dem schizophrenen Formenkreis war im Laufe ihres Lebens Gewalt ausgesetzt. Neben anderen Folgen liegt bei einem Teil der Betroffenen auch eine komorbide PTBS vor. Gewalterfahrungen und ihre Folgen sollten deshalb routinemäßig erhoben und das gesamte Spektrum traumatherapeutischer Ansätze in die Behandlung von Patientinnen und Patienten mit Störungen aus dem schizophrenen Formenkreis integriert werden. 


\section{Fazit für die Praxis}

- Die Hälfte der weiblichen und ein Drittel der männlichen Patienten mit Störungen aus dem schizophrenen Formenkreis waren in ihrer Kindheit sexueller und/oder körperlicher Gewalt ausgesetzt

- Zwei Drittel aller Patienten mussten irgendwann in ihrem Leben Gewalterfahrungen machen.

- Weibliche Patientinnen waren sowohl in der Kindheit als auch im Erwachsenenalter häufiger sexueller Gewalt ausgesetzt, männliche Patienten im Erwachsenenalter häufiger körperlicher Gewalt.

- Besonders Gewalt in der Kindheit kann bei schizophrenen Patienten mit einem Spektrum von Folgen assoziiert sein, wie Störungen der Emotionsregulation, interpersonellen Problemen und komorbiden Posttraumatischen Belastungsstörungen, die das klinische Bild zusätzlich erschweren und einer spezifischen Behandlung bedürfen.

- Die Folgen von Gewalt können mit Methoden aus dem Bereich der Traumatherapie effektiv behandelt werden, die sich inzwischen auch bei Patienten mit Störungen aus dem schizophrenen Formenkreis bewährt haben, bei dieser Patientengruppe jedoch bislang zu selten eingesetzt werden.

\section{Einhaltung ethischer Richtlinien}

Die Autoren geben an, dass keine Interessenkonflikte bestehen.

Die Untersuchung wurde mit Zustimmung der zuständigen Ethik-Kommission durchgeführt. Von allen beteiligten Patienten wurde ihr schriftliches Einverständnis zur Teilnahme eingeholt. 


\section{Literatur}

1. Assion H-J, Brune N, Schmidt N, Aubel T, Edel M-E, Basilowski M, Juckel G, Frommberger $U$ (2009) Trauma exposure and post-traumatic stress disorder in bipolar disorder. Soc Psychiat Epidemiol 44:1041-1049

2. Bengtson-Tops A, Ehliasson $K$ (2011) Victimization in individuals suffering from psychosis: a Swedish cross-sectional study. Journal of Psychiatric and Mental Health Nursing: 1-8

3. Bieneck S, Stadler L, Pfeiffer C (2011) Erster Forschungsbericht zur Repräsentativbefragung Sexueller Missbrauch 2011. Hannover: Kriminologisches Forschungsinstitut Niedersachsen

4. Bonoldi I, Simeone E, Rocchetti M, et al. (2013) Prevalence of self-reported childhood abuse in psychosis: A meta-analysis of retrospective studies. Psychiatry Research 210: 8-15

5. Cloitre M, Cohen LR, Koenen KC (2014) Sexueller Missbrauch und Misshandlung in der Kindheit. Ein Therapieprogramm zur Behandlung komplexer Traumafolgen. Göttingen: Hogrefe-Verlag

6. Conus P, Cotton S, Schimmelmann BG, McGorry PD, Lambert M (2010) Pretreatment and outcome correlates of sexual and physical trauma in an epidemiological cohort of first-episode psychosis patients. Schizophr Bull 36:1105-1114

7. Dean K, Moran P, Fahy T, Tyrer P, Leese M, Creed F, Burns T, Murray R, Walsh E (2007). Predictors of violent victimization amongst those with psychosis. Acta Psychiatr Scand 116: 345-353

8. Draijer N (1989) Structured Trauma Interview. Amsterdam: Department of Psychiatry, Free University

9. Fallot RD, Harris M (2002) The Trauma Recovery and Empowerment Model (TREM): Conceptual and practical issues in a group intervention for women. Community Mental Health Journal 38, 475-485

10. First MB, Spitzer RL, Williams JBW, Gibbon M (1997) Structured Clinical Interview for DSM-IV Axis I Disorders (SCID-I), Clinician Version. Arlington, Virginia, American Psychiatric Publishing Inc.

11. Fisher HL, Craig TK, Fearon P, Morgan K, Dazzan P, Lappin J, Hutchinson G, Doody GA Jones PB, McGuffin P, Murray RM, Leff J, Morgan C (2011) Reliability 
and comparability of psychosis patient's retrospective reports of childhood abuse. Schizophr Bull 37:546-553

12. Hammer JH, Springer J, Beck NC, Menditto A, Coleman J (2011) The relationship between seclusion and restraint use and childhood abuse among psychiatric inpatients. J Interpers Violence 26:567-579

13. Kay SR, Fiszbein A, Opler LA (1987) The positive and negative syndrome scale (PANSS) for schizophrenia. Schizophr Bull 13:261-276

14. Langeland W, Draijer N, van den Brink W (2004) Psychiatric comorbidity in treatment-seeking alcoholics: The role of childhood trauma and perceived parental dysfunction. Alcohol Clin Exp Res 28:441-447

15. Lecrubier Y, Sheehan D, Weiller E, Amorim P, Bonora I, Sheehan K, Janavs, J, Dunbar GC (1997) The MINI International Neuropsychiatric Interview (M.I.N.I), a short diagnostic interview: Reliability and validity according to the CIDI. Eur Psychiatry 12:224-331

16. Lysaker PH, Nees MA, Lancaster RS, Davis LW (2004) Vocational function among persons with schizophrenia with and without history of childhood sexual trauma. J Trauma Stress 17:435-438

17. Lysaker PH, Salyers MP (2007) Anxiety symptoms in schizophrenia spectrumdisorders: associations with social function, positive and negative symptoms, hope and trauma history. Acta Psychiatr Scand 116:290-298

18. Morgan C., Fisher H (2007) Environment and schizophrenia: Environmental factors in schizophrenia: childhood trauma - a critical review. Schizophr Bull 33:3-10

19. Mueser KT, Rosenberg SD, Goodman LA, Trumbetta SL (2002) Trauma, PTSD, and the course of severe mental illness: an interactivemodel. Schizophr Res 53:123-143

20. Mueser KT, Rosenberg SD, Rosenberg HJ (2009) Treatment of Posttraumatic Stress Disorder in special populations. A cognitive restructuring program. Washington DC: American Psychological Association

21. Roelofs K, Keijsers GPJ, Hoogduin KAL, Näring GWB, Moene FC (2002) Childhood abuse in patients with conversion disorder. Am J Psychiatry 159:19081913 
22. Schäfer I, Fischer HL (2011) Childhood trauma and PTSD in patients withpsychosis - clinical challenges and emerging treatments. Curr Opin Psychiatry 24:514-518

23. Schäfer I, Morgan C, Demjaha A, Morgan K, Dazzan P, Fearon P, Jones PB, Doody GA, Leff J, Murray RM, Fisher HL (2011) Assessment of posttraumatic symptoms in patients with first-episode psychosis. J Nerv Ment Dis 199:896-898

24. Shah S, Mackinnon A, Galletly C, et al. (2014) Prevalence and impact of childhood abuse in people with a psychotic illness. Data from the second Australian national survey of psychosis. Schiz Res 159: 20-26

25. Shannon C, Douse K, McCusker C, Feeney L, Barrett S, Mulholland C (2009) The association between childhood trauma and memory functioning in schizophrenia. Schizophr Bull 37:531-537

26. Spitzer C, Barnow S, Volzke H, John U, Freyberger HJ, Grabe HJ (2009) Trauma, posttraumatic stress disorder, and physical illness: findings from the general population. Psychosom Med 71(9):1012-1017

27. Trappler B, Newville H (2007) Trauma healing via cognitivebehaviortherapy in chronicallyhospitalizedpatients. Psychiatr Q 78:317-325

28. Van den Berg DP, de Bont PA, van der Vleugel BM, de Roos C, de Jongh A, Van Minnen A, van der Gaag M (2015) Prolonged Exposure vs Eye Movement Desensitization and Reprocessing vs Waiting List for Posttraumatic Stress Disorder in Patients With a Psychotic Disorder: A Randomized Clinical Trial. JAMA Psychiatry 72:259-267

29. Vogel M, Spitzer C, Barnow S, Freyberger HJ, Grabe HJ (2009) Association of Childhood Neglect with Adult Dissociation in inpatients with Schizophrenia. Psychopathology 42(2):124-130

30. Wetzels P (1997) Zur Epidemiologie physischer und sexueller Gewalterfahrungen in der Kindheit. Ergebnisse einer repräsentativen Prävalenzstudie für die BRD. Kriminologisches Forschungsinstitut Niedersachsen, Forschungsberichte Nr. 59 
Tabelle 1. Sexuelle und körperliche Gewalterfahrungen in unterschiedlichen Lebensphasen (Strukturiertes Traumainterview; [8])

\begin{tabular}{|c|c|c|c|c|c|}
\hline & & $\begin{array}{c}\text { Weiblich } \\
N(\%)\end{array}$ & $\begin{array}{c}\text { Männlich } \\
N(\%)\end{array}$ & $\begin{array}{c}\text { Gesamt } \\
N(\%)\end{array}$ & $p$-Wert \\
\hline Gewalt & sexuell & $13(27)$ & $12(12)$ & $25(17)$ & $p=0,027$ \\
\hline \multirow[t]{3}{*}{ Kindheit } & körperlich & $18(38)$ & $28(29)$ & $46(32)$ & $p=0,293$ \\
\hline & beides & $8(17)$ & $7(7)$ & $15(10)$ & $p=0,079$ \\
\hline & mind. eine Form & $23(48)$ & $33(34)$ & $56(39)$ & $p=0,106$ \\
\hline Gewalt & sexuell & $14(30)$ & $10(10)$ & $24(17)$ & $p=0,004$ \\
\hline \multirow[t]{3}{*}{ Erwachsenenalter } & körperlich & $13(27)$ & $43(44)$ & $56(39)$ & $p=0,045$ \\
\hline & beides & $5(10)$ & $7(7)$ & $12(8)$ & $p=0,510$ \\
\hline & mind. eine Form & $24(50)$ & $46(47)$ & $70(48)$ & $p=0,770$ \\
\hline Gewalt & sexuell & $23(48)$ & $20(21)$ & $43(30)$ & $p=0,001$ \\
\hline \multirow[t]{3}{*}{ Lebenszeit } & körperlich & $22(46)$ & $57(59)$ & $79(55)$ & $p=0,141$ \\
\hline & beides & $11(23)$ & $12(12)$ & $23(16)$ & $p=0,102$ \\
\hline & mind. eine Form & $34(71)$ & $62(64)$ & $96(66)$ & $p=0,407$ \\
\hline
\end{tabular}

Anmerkungen. Weiblich: $\mathrm{N}=48$; Männlich: $\mathrm{N}=97$; signif. Geschlechtsunterschieden sind $p$-Werte sind durch Fettdruck hervorgehoben 